\title{
IMPACTO DE PROGRAMAS DE GERENCIAMENTO DA DEMANDA NO CUSTO DA PERDA DE CARGA
}

\author{
Luiz A. F. Manso* \\ manso@mgconecta.com.br \\ Armando M. Leite da Silva ${ }^{\dagger}$ \\ armando@iee.efei.br \\ *Departamento de Eletricidade - DEPEL, Universidade Federal de São João del-Rei - UFSJ \\ ${ }^{\dagger}$ Grupo de Engenharia de Sistemas - GESis, Universidade Federal de Itajubá - UNIFEI
}

\section{ABSTRACT}

This paper presents an application of pseudochronological Monte Carlo simulation algorithm to investigate the effects of load management on interruption cost. The pseudo-chronological simulation, which retains the computational efficiency of non-sequential simulation and the accuracy of chronological simulation, can be used to represent different load patterns per area or bus. Case studies on the Brazilian SouthSoutheastern System (SSB) considering the impact of different load management policies applied to the Rio de Janeiro area are presented and discussed.

KEYWORDS: Load management, demand-side management, Monte Carlo simulation, composite reliability, uncertainty modeling, interruption costs.

\section{RESUMO}

Este artigo descreve uma aplicação da simulação Monte Carlo pseudo-cronológica em estudos de impacto do gerenciamento da demanda no custo da perda de carga. A simulação pseudo-cronológica apresenta a eficiência computacional da simulação não-seqüencial e a precisão da simulação cronológica, podendo ser utilizada para representar diferentes padrões comportamentais para as cargas das diversas áreas ou barras de um sistema. O

Artigo submetido em $18 / 12 / 2000$

1a. Revisão em 28/4/2003

Aceito sob recomendação do Ed. Assoc. Prof. Glauco N. Taranto sistema SSB (Sistema Sul-Sudeste Brasileiro) é utilizado para testes, sendo avaliados os impactos obtidos por diferentes políticas de gerenciamento da carga na área Rio.

PALAVRAS-CHAVE: Gerenciamento da demanda, simulação Monte Carlo, confiabilidade composta, modelagem de incertezas, custos de interrupção.

\section{INTRODUÇÃO}

Os programas de gerenciamento da demanda têm como objetivo alterar o padrão da curva de carga de modo a reduzir as variações entre seus picos e vales. Como em um sistema de potência a energia elétrica deve ser produzida e consumida ao mesmo tempo, é necessário haver um deslocamento cronológico de determinadas cargas. Este deslocamento pode ocorrer no sentido dos horários de pico para os horários de cargas mais leves, em um determinado dia, ou ainda, no sentido de dias úteis para os finais de semana.

A redução dos custos operativos do sistema e a postergação de investimentos (Gellings e Smith, 1989; Gaul et alii, 1998; Malik, 1998) têm sido apresentadas como os principais benefícios de programas de gerenciamento da demanda. Porém, um outro aspecto, não menos relevante, é o impacto destes programas nos custos de interrupção do fornecimento de energia, ou custo da perda de carga. O índice LOLC (loss of load cost) corresponde ao valor esperado do custo da perda de carga. Para 
uma avaliação precisa deste índice é necessário caracterizar corretamente o processo de interrupção de energia, identificando as durações associadas a todos os blocos de energia não suprida, em cada barra e para cada classe consumidora (Leite da Silva et alii, 1997; Manso et alii, 1998; Manso et alii, 1999 a). Desta forma, a decisão de escolher um determinado programa de gerenciamento de demanda deve levar em conta os diversos custos envolvidos, incluindo a LOLC.

Para melhor avaliar os benefícios obtidos através do gerenciamento da demanda, os algoritmos de simulação utilizados devem adotar uma representação detalhada da carga, capaz de considerar diferentes padrões de carga para as diversas áreas ou barras do sistema. A importância da modelagem de cargas variantes no tempo é discutida em (Sankarakrishnan e Billinton, 1995; Mello et alii, 1997 b; Manso et alii, 1999 b; Leite da Silva et alii, 2000).

A simulação Monte Carlo seqüencial é uma ferramenta natural para simular aspectos cronológicos, e portanto, capaz de representar cargas variantes no tempo. Também as durações dos blocos de energia interrompida podem ser identificadas de maneira precisa. A modelagem cronológica requer esforço computacional substancialmente maior que aquele apresentado pelas técnicas não-seqüenciais (Singh et alii, 1993; Manso et alii, 1999 a). Já a simulação pseudo-cronológica (Manso et alii, 1999 b; Leite da Silva et alii, 2000), embora baseada em modelos de Markov, mantém um elevado grau de flexibilidade, sendo capaz de representar diferentes padrões de carga por área ou barra do sistema. Esta nova técnica mantém a precisão da simulação Monte Carlo seqüencial, porém requer um esforço computacional semelhante ao dos algoritmos não-seqüenciais.

Este artigo apresenta uma aplicação da simulação Monte Carlo pseudo-cronológica em estudos de impacto do gerenciamento da demanda no custo da perda de carga. O sistema SSB (Sistema Sul-Sudeste Brasileiro) é utilizado para testes, sendo avaliados os impactos obtidos pelo gerenciamento da carga da área Rio.

\section{SIMULAÇÃO PSEUDO-CRONOLÓGI- CA}

A simulação Monte Carlo pseudo-cronológica é um novo método (Manso et alii, 1999 b; Leite da Silva et alii, 2000), o qual combina a eficiência computacional da simulação não-seqüencial com a habilidade de modelar curvas de carga apresentada pela simulação Monte Carlo seqüencial.

\subsection{Modelo de Markov Não-Agregado com Múltiplos Níveis}

Qualquer modelo de carga irá reproduzir de maneira aproximada o seu real comportamento. A precisão de cada modelo depende da quantidade e qualidade de dados disponíveis. Modelos de Markov podem ser verificados não apenas para o processo de falha e reparo de equipamentos, mas também para o comportamento da carga. Usualmente curvas cronológicas de carga com 8760 pontos horários são disponíveis e fornecem informações extremamente úteis aos estudos de confiabilidade de sistemas de potência (Melo et alii, 1993).

Usando as hipóteses de Markov, as curvas horárias da carga são transformadas em modelos multi-estados, não balanceados em freqüência. Para reduzir o número de estados a serem analisados, são utilizadas técnicas de agrupamento (clustering techniques) (Anderberg, 1973). Este processo de agregação implica na redução do espaço amostral, sendo vital apenas para o método da enumeração de estados. Também é importante destacar que os modelos de Markov agregados para a carga não são capazes de representar cargas variantes no tempo.

Se os componentes do sistema são não-Markovianos e se cargas variantes no tempo são especificadas por área, barra ou até mesmo por classe consumidora, a única opção para avaliar índices de confiabilidade é através da simulação Monte Carlo seqüencial. O preço a ser pago por adotar uma representação tão detalhada, é um tempo de CPU extremamente elevado. Em se tratando de sistemas de grande porte, esta avaliação pode se tornar inviável. Tendo em mente esta restrição, e considerando que as cargas podem variar no tempo segundo padrões identificados para cada área, foi proposto o modelo de Markov não-agregado com múltiplos níveis descrito pela Figura 1. Para manter algumas informações cronológicas a respeito da carga, o modelo é composto por um conjunto de $T$ níveis múltiplos de carga, conectados na mesma ordem em que aparecem no histórico da carga.

O modelo utiliza uma taxa de transição constante $\lambda_{L}=$ $1 / \Delta T$, onde $\Delta T$ representa a unidade de tempo usada para discretizar o período $T$. Para cada uma das $m$ áreas consideradas, é fornecido o nível de carga por intervalo de tempo. Como exemplo, $L_{h}\left(A_{m}\right)$ corresponde ao nível de carga da hora $h$ apresentado pela área $m$. Como demonstra a Figura 1, quando a carga da área 1 transita do nível 1 para o nível 2, i.e. de $L_{1}\left(A_{1}\right)$ para $L_{2}\left(A_{1}\right)$, o mesmo ocorre para as demais áreas, ou seja, $L_{1}\left(A_{2}\right)$ transita para $L_{2}\left(A_{2}\right), \ldots$ e $L_{1}\left(A_{m}\right)$ transita para $L_{2}\left(A_{m}\right)$. Visto que todas as taxas de transição são iguais, as cargas permanecerão, em média, $\Delta T$ ho- 


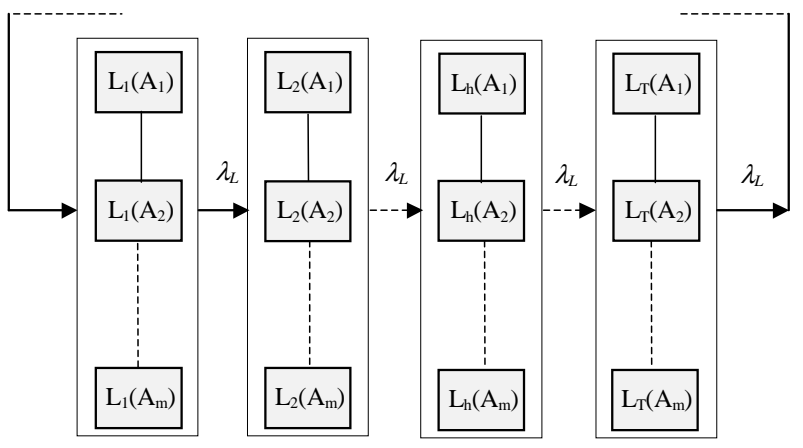

Figura 1: Modelo de Markov Não-Agregado com Múltiplos Níveis

ras (e.g. 1 hora) em cada nível, assim como o período de análise terá, em média, $T$ horas (e.g. 8760 horas). Obviamente, o conceito de área pode ser estendido para barra ou classe consumidora.

Certamente o modelo de carga proposto é extremamente flexível e superior à maioria dos modelos de Markov discutidos pela literatura. A principal vantagem deste modelo está em manter, de maneira aproximada, a representação cronológica. Em média, o estado $h$ do modelo corresponderá à hora $h$ da curva cronológica da carga. Através do modelo proposto, a avaliação de índices de confiabilidade composta fica mais flexível: não é mais necessário assumir coerência para o sistema, assim como é possível considerar cargas variantes no tempo. Note que, até mesmo políticas de manutenção associadas ao tempo podem ser implementadas de maneira aproximada.

\subsection{Estimação dos Índices}

Através da simulação Monte Carlo, os índices de perda de carga podem ser estimados como a média de $N$ amostras da função teste $F\left(x^{k}\right)$, i.e. (Melo et alii, 1993; Mello et alii, 1994):

$$
\tilde{E}[F]=1 / N \sum_{k=1}^{N} F\left(x^{k}\right)
$$

Utilizando uma função teste adequada, qualquer índice básico de confiabilidade pode ser estimado via Eq. (1), cuja incerteza é dada pela variância do estimador:

$$
V(\tilde{E}[F])=V(F) / N
$$

onde $V(F)$ é a variância da função teste. Esta incerteza é usualmente representada como um coeficiente de variação (Melo et alii, 1993):

$$
\beta=\sqrt{V(\tilde{E}[F])} / \tilde{E}[F]
$$

\subsection{1 Índices de probabilidade e energia}

Através das funções teste $F_{L O L P}$ e $F_{E E N S}$, dadas a seguir, a simulação não-seqüencial fornece estimativas não-tendenciosas para os índices LOLP (loss of load probability) e EENS (expected energy not supplied).

$$
F_{\text {LOLP }}\left(x^{k}\right)= \begin{cases}0 & \text { se } x^{k} \in X_{\text {Sucesso }} \\ 1 & \text { se } x^{k} \in X_{\text {Falha }}\end{cases}
$$

e

$$
F_{\text {EENS }}\left(x^{k}\right)= \begin{cases}0 & \text { se } x^{k} \in X_{\text {Sucesso }} \\ \Delta P_{k} \times T & \text { se } x^{k} \in X_{\text {Falha }}\end{cases}
$$

onde $X=X_{\text {Sucesso }} \cup X_{\text {Falha }}$ é o conjunto de todas as possíveis realizações de $x$ (i.e. o espaço de estados), divido em dois subespaços $X_{\text {Sucesso }}$ (estados de sucesso) e $X_{\text {Falha }}$ (estados de falha); $\Delta P_{k}$ é o corte de potência aplicado no estado de falha $x^{k}$ e $T$ é o período de análise.

Observe que as expressões (4) e (5) dependem somente das distribuições de probabilidade associadas aos estados do sistema $x^{k}$, que por sua vez dependem das distribuições de probabilidade dos equipamentos e da carga. Portanto, a estimação de índices de probabilidade e de energia, utilizando a parcela não-seqüencial do algoritmo da simulação pseudo-cronológica, é precisa e extremamente rápida.

\subsection{2 Índices de freqüência e duração}

Uma pequena variação da simulação pseudo-seqüencial (Mello et alii, 1994), combinando o método da transição de estados (Kumamoto et alii, 1980; Billinton e Li, 1993) e a simulação Monte Carlo não-seqüencial, foi proposta recentemente (Mello et alii, 1997 a). Partindo de um estado de falha $\left(x^{k}\right)$ amostrado via sorteio nãoseqüencial, a interrupção é corretamente caracterizada através da chamada simulação forward/backward. A duração $D_{I}$, de uma interrupção $I$, é dada pela soma das durações $D^{i}$ associadas aos estados de falha $x^{i}$, os quais formam a seqüência de interrupção.

Até mesmo quando cargas variantes no tempo são consideradas, estimativas não-tendenciosas para a LOLF (loss of load frequency) podem ser obtidas utilizandose o modelo da Figura 1 em conjunto com a seguinte função teste:

$$
F_{\text {LOLF }}\left(x^{k}\right)= \begin{cases}0 & \text { se } x^{k} \in X_{\text {Sucesso }} \\ 1 / E\left[D_{I}\right] & \text { se } x^{k} \in X_{\text {Falha }}\end{cases}
$$


O índice LOLD (loss of load duration) pode ser calculado através da seguinte equação:

$$
L O L D=L O L P / L O L F
$$

\subsection{3 Índices de custo de interrupção}

A quantidade de energia interrompida (kWh) e o custo unitário de interrupção (US\$/kWh), são os aspectos mais relevantes para estimar o impacto de uma interrupção. Pesquisas diretas aos consumidores indicam que o custo unitário de interrupção depende de fatores como duração, freqüência, instante de ocorrência, notificação prévia, montante cortado e abrangência geográfica (EPRI, 1989; Wacker e Billinton, 1989). Dentre eles, o mais importante é a duração da interrupção. Portanto, é necessário utilizar a representação cronológica, ao menos para a falha.

Ao considerar cargas variantes no tempo, a estimativa para a LOLC é obtida utilizando-se o modelo de carga da Figura 1 e a seguinte função teste não-tendenciosa:

$$
F_{\text {LOLC }}\left(x^{k}\right)= \begin{cases}0 & \text { se } x^{k} \in X_{\text {Sucesso }} \\ K_{I} / E\left[D_{I}\right] & \text { se } x^{k} \in X_{\text {Falha }}\end{cases}
$$

onde $K_{I}$ é o custo, em US\$, de uma interrupção $I$, amostrada a partir da mesma simulação forward/backward, já utilizada para a obtenção de índices de freqüência e duração.

\subsection{Algoritmo}

O algoritmo da simulação pseudo-cronológica é implementado através dos seguintes passos:

(i) amostre um estado do sistema $x^{k} \in X$, baseado em sua distribuição de probabilidade $P(x)$;

(ii) analise o desempenho do estado amostrado $x^{k}$ por meio de estudos de fluxo de potência. Se necessário, acione medidas corretivas. Se $x^{k}$ é um estado de sucesso retorne ao passo (i); senão, estime funções teste para os índices LOLP (Eq. 4) e EENS (Eq. 5) e prossiga no passo (iii);

(iii) obtenha através da simulação forward/backward, uma seqüência de interrupção $I$ associada com o estado de falha $x^{k}$. Estime funções teste para os índices LOLF (Eq. 6) e LOLC (Eq. 8);

(iv) avalie estimativas para os índices (Eq. 1) e os respectivos coeficientes de variação (Eq. 3). Se a convergência desejada não é obtida, retorne ao passo (i); caso contrário, avalie o índice LOLD (Eq. 7) e pare.

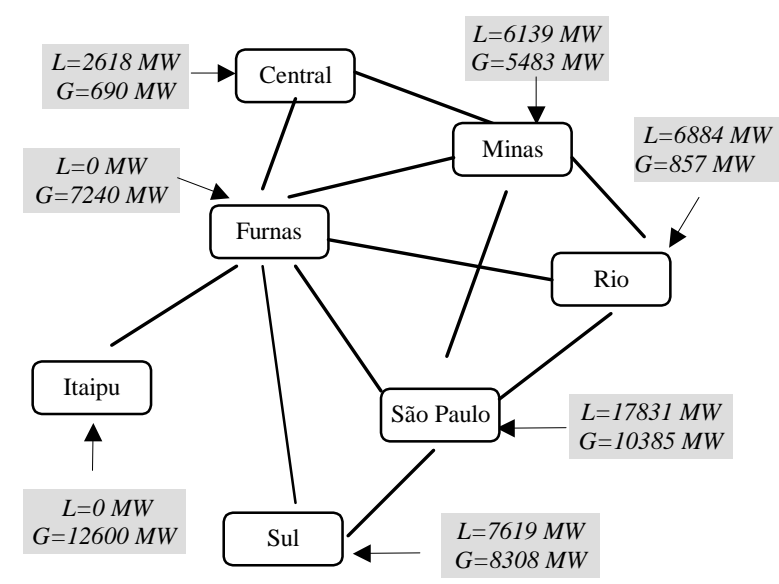

Figura 2: Diagrama Simplificado do Sistema SSB

\section{CASOS ESTUDADOS}

Os casos estudados referem-se ao gerenciamento da demanda da área Rio. Para tal, foi utilizada uma versão reduzida do sistema Sul-Sudeste Brasileiro - SSB, a qual é composta por 413 barras e 685 circuitos. A capacidade instalada e o pico de carga são aproximadamente iguais a $46 \mathrm{GW}$ e $41 \mathrm{GW}$, respectivamente. A Figura 2 resume as informações básicas através de um diagrama simplificado ( $L=$ carga e $G=$ geração da área) do sistema estudado. As curvas de custo unitário de interrupção foram extraídas de pesquisas realizadas pela Eletrobrás (Massaud et alii, 1992). A composição da carga global do sistema é $19 \%$ residencial, $28 \%$ comercial e $53 \%$ industrial.

Para representar o comportamento da carga, nas cinco áreas (São Paulo, Minas, Rio, Sul e Centro), são utilizadas 5 curvas de carga com 8736 pontos horários. Existem fatores de carga diários, semanais e mensais, para cada uma das áreas de carga. A Figura 3 apresenta curvas típicas diárias (Sexta e Sábado) no verão e inverno para a área Rio. O comportamento distinto da carga é devido principalmente às condições mais severas durante o verão.

Considerando que os horários de carga pico, para a área Rio, são diferentes para inverno e verão, foram definidas duas estratégias para a redução da ponta diária de carga: uma para os meses de "inverno" (Abr-Set) e outra para os meses de "verão" (Out-Mar). Através das curvas típicas para dias úteis de inverno e verão é possível identificar que os respectivos picos ocorrem para os períodos de 17 às 21 horas e de 13 às 17 horas. 


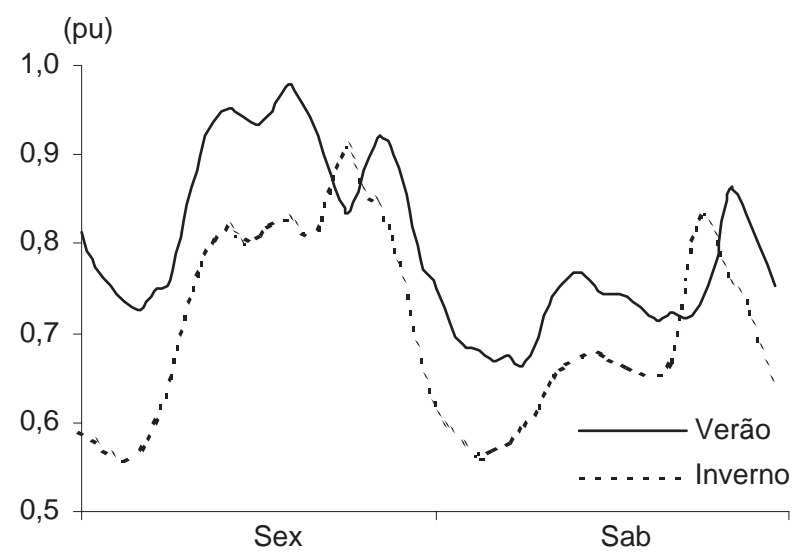

Figura 3: Curvas Diárias Típicas - Área Rio

A variedade de padrões de carga ilustra a importância de uma detalhada representação para a cronologia da carga ou, ao menos, de um modelo de carga a espaço de estados, hábil para considerar os diferentes padrões da carga. Esta representação é indispensável para a avaliação de programas de gerenciamento da demanda, quando se está especificamente alterando o padrão de determinadas cargas.

Seis estudos de caso foram considerados de modo a variar a redução do pico diário de carga em profundidade (pu) e em amplitude (horas). Com relação ao destino destas cargas, foram utilizadas duas políticas. Na Política 1 as cargas são deslocadas para o período compreendido entre 7 e 13 horas dos dias úteis. Na Política 2, o deslocamento é feito para os sábados, no período de 8 às 18 horas. Esta última política pode ser entendida como o resultado de dois processos de deslocamento de carga: transferência de cargas, de todos os setores, do horário de pico para o período fora da ponta, realizado em dias úteis, e transferência de cargas industriais de dias úteis para os sábados. Os seis casos estudados são descritos pelas Tabelas 1 e 2, onde, na segunda coluna, os períodos superiores ( são referentes aos meses de

Tabela 1: Política 1: Destino para Dias Úteis

\begin{tabular}{|c|c|c|c|c|}
\hline Caso & \multicolumn{2}{|c|}{ Deslocado de } & \multicolumn{2}{c|}{ Para } \\
& \multicolumn{2}{|c|}{ Período(h) } & Carga(\%) & \multicolumn{2}{c|}{ Período(h) } & Carga(\%) \\
\hline 1 & $\begin{array}{c}17 \text { às } 19^{\star} \\
14 \text { às } 16^{\star}\end{array}$ & 5,00 & 7 às 13 & 1,67 \\
\hline 2 & $\begin{array}{c}17 \text { às } 19^{\star} \\
14 \text { às } 16^{\star}\end{array}$ & 10,00 & 7 às 13 & 3,33 \\
\hline 3 & $\begin{array}{c}17 \text { às } 21^{\star} \\
13 \text { às } 17^{\star}\end{array}$ & 5,00 & 7 às 13 & 3,33 \\
\hline
\end{tabular}

Tabela 2: Política 2: Destino para os Sábados

\begin{tabular}{|c|c|c|c|c|}
\hline \multirow[t]{2}{*}{ Caso } & \multicolumn{2}{|c|}{ Deslocado de } & \multicolumn{2}{|c|}{ Para } \\
\hline & Período $(\mathrm{h})$ & Carga $(\%)$ & Período $(\mathrm{h})$ & Carga $(\%)$ \\
\hline 1 & $\begin{array}{l}17 \text { às } 19^{*} \\
14 \text { às } 16^{\star}\end{array}$ & 5,00 & 8 às 18 & 5,00 \\
\hline 2 & $\begin{array}{l}17 \text { às } 19^{*} \\
14 \text { às } 16^{\star}\end{array}$ & 10,00 & 8 às 18 & 10,00 \\
\hline 3 & $\begin{array}{l}17 \text { às } 21^{\text {* }} \\
13 \text { às } 17^{\star}\end{array}$ & 5,00 & 8 às 18 & 10,00 \\
\hline
\end{tabular}

"inverno", enquanto que os períodos inferiores $\left(^{\star}\right)$ são aplicados aos meses de "verão".

A Tabela 3 apresenta, para as diversas áreas e sistema, a energia esperada não suprida em GWh/ano e em valores percentuais relativos ao consumo anual de energia, obtidas para o caso base, i.e., sem alterações na curva de carga da área Rio. O valor esperado do custo de interrupção (LOLC) também é fornecido nesta tabela, por exemplo, 45,060 milhões de dólares por ano para o sistema.

Os custos de interrupção obtidos para os seis casos estudados são apresentados pelas Tabelas 4 e 5 . São incluídas, entre parênteses, as diferenças percentuais em relação ao caso base. Através dos resultados obtidos pode-se concluir que o impacto da política 2 é significativamente maior. Para o Caso 6, que apresentou o melhor resultado, a redução na LOLC foi de $4,344 \times 10^{6}$ US\$/ano, contra um ganho de $2,160 \times 10^{6}$ US $\$$ /ano obtido pelo caso análogo na política 1 (Caso 3). Do ponto de vista do custo de interrupção, pode-se afirmar que, dentro da política 1, não é aconselhável aprofundar o volume de carga transferido, pois a LOLC do Caso 2 é maior que a LOLC do Caso 1. Já a ampliação de seu período (Caso3) produz resultados positivos, embora pouco significativos. Em relação à política 2, vê-se que a opção mais vantajosa é ampliar o período de redução do pico de carga (Caso 6).

Portanto, a melhor política consiste em reduzir o pico diário da carga, em um período mais dilatado (4 ho-

Tabela 3: Índices Estimados para o Caso Base

\begin{tabular}{|c|c|c|c|}
\hline Área & \multicolumn{2}{|c|}{ EENS } & LOLC \\
& $(\mathrm{GWh} / \mathrm{a})$ & $(\%)$ & $\left(10^{6}\right.$ US $\left.\$ / \mathrm{a}\right)$ \\
\hline Rio & 11,671 & 0,0254 & 17,236 \\
\hline São Paulo & 1,5581 & 0,0014 & 2,7279 \\
\hline Minas & 2,6391 & 0,0064 & 6,4691 \\
\hline Sul & 6,3395 & 0,0146 & 11,072 \\
\hline Central & 3,5114 & 0,0251 & 7,5550 \\
\hline Sistema & 25,719 & 0,0099 & 45,060 \\
\hline
\end{tabular}


Tabela 4: Índice LOLC para os Casos 1, 2 e 3

\begin{tabular}{|c|c|c|c|}
\hline Área & \multicolumn{3}{|c|}{ LOLC $\left(10^{6}\right.$ US\$/ano) } \\
& Caso 1 & Caso 2 & Caso 3 \\
\hline Rio & 15,783 & 16,248 & 15,554 \\
& $(-8,43 \%)$ & $(-5,73 \%)$ & $(-9,76 \%)$ \\
\hline São Paulo & 2,5951 & 2,6317 & 2,5528 \\
& $(-4,87 \%)$ & $(-3,53 \%)$ & $(-6,42 \%)$ \\
\hline Minas & 6,4601 & 6,4820 & 6,4764 \\
& $(-, 14 \%)$ & $(, 20 \%)$ & $(, 11 \%)$ \\
\hline Sul & 10,799 & 10,659 & 10,741 \\
& $(-2,47 \%)$ & $(-3,73 \%)$ & $(-2,99 \%)$ \\
\hline Central & 7,5312 & 7,5194 & 7,5759 \\
& $(-, 32 \%)$ & $(-, 47 \%)$ & $(, 28 \%)$ \\
\hline Sistema & 43,168 & $43,540)$ & 42,900 \\
& $(-4,20 \%)$ & $(-3,37 \%)$ & $(-4,79 \%)$ \\
\hline
\end{tabular}

Tabela 5: Índice LOLC para os Casos 4, 5 e 6

\begin{tabular}{|c|c|c|c|}
\hline Área & \multicolumn{3}{|c|}{ LOLC $\left(10^{6}\right.$ US $\$ /$ ano $)$} \\
& Case 4 & Case 5 & Case 6 \\
\hline Rio & 14,927 & 14,263 & 13,483 \\
& $(-13,40 \%)$ & $(-17,25 \%)$ & $(-21,77 \%)$ \\
\hline São Paulo & 2,5956 & 2,6333 & 2,5546 \\
& $(-4,85 \%)$ & $(-3,47 \%)$ & $(-6,35 \%)$ \\
\hline Minas & 6,3770 & 6,3606 & 6,3657 \\
& $(-1,42 \%)$ & $(-1,68 \%)$ & $(-1,60 \%)$ \\
\hline Sul & 10,798 & 10,654 & 10,737 \\
& $(-2,47 \%)$ & $(-3,78 \%)$ & $(-3,03 \%)$ \\
\hline Central & 7,5317 & 7,5198 & 7,5762 \\
& $(-, 31 \%)$ & $(-, 47 \%)$ & $(, 28 \%)$ \\
\hline Sistema & 42,229 & 41,431 & 40,716 \\
& $(-6,28 \%)$ & $(-8,05 \%)$ & $(-9,64 \%)$ \\
\hline
\end{tabular}

ras), sem contudo elevar o consumo nas demais horas dos dias úteis. Isto implica no deslocamento de cargas de dias úteis para os sábados. Através desta política, Caso 6, a redução da LOLC para a área Rio (onde foi adotado o gerenciamento da demanda) foi de $3,753 \times 10^{6}$ US\$/ano, representando um ganho superior a $21,7 \%$ do valor original.

As Figuras 4 e 5 apresentam, respectivamente para verão e inverno, as curvas originais e modificadas pelo gerenciamento da demanda, Caso 6, na área Rio.

Finalmente, a Tabela 6 mostra a variação dos índices estimados para o Caso Base, sem gerenciamento da demanda, e para o Caso 6, que demonstrou ser a melhor política de gerenciamento da demanda entre as analisadas. Há reduções relevantes nos índices do sistema EENS e LOLC, $11 \%$ e 9,6\%, respectivamente, enquanto que os demais índices apresentam reduções marginais de 1 a $2 \%$. Estes resultados demonstram que a probabilidade e freqüência do corte, para o sistema, permanecem

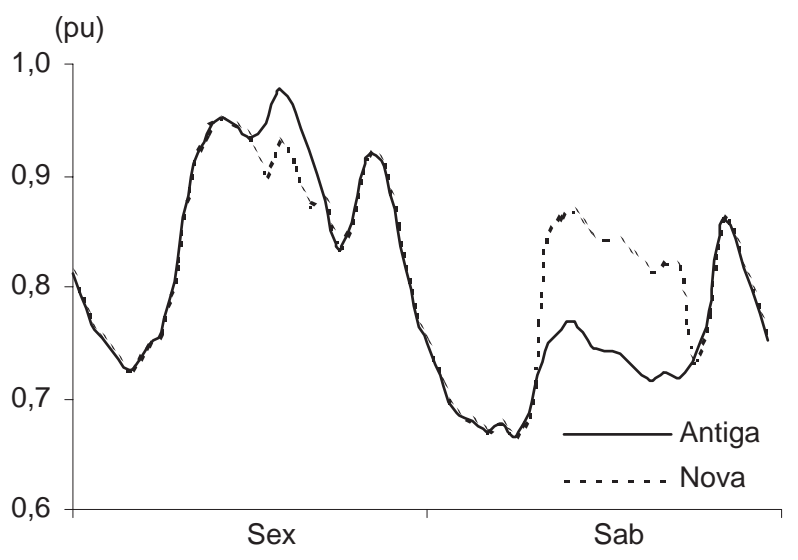

Figura 4: Curva Típica para o Verão - Área Rio

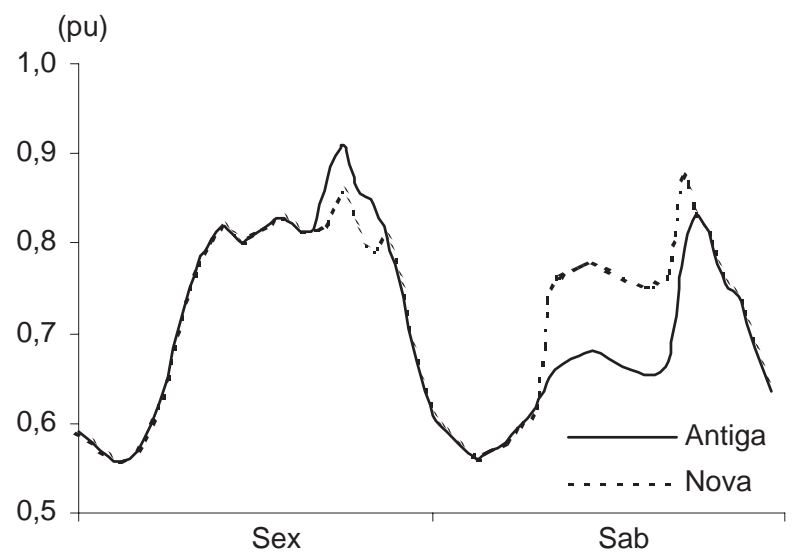

Figura 5: Curva Típica para o Inverno - Área Rio

basicamente inalteradas. Portanto, os ganhos obtidos se devem, em grande parte, a uma redução no aprofundamento do corte de carga. Obviamente, a probabilidade e freqüência do corte foi reduzida em diversas barras da área Rio.

Tabela 6: Índices Estimados para o Sistema

\begin{tabular}{|c|c|c|c|}
\hline Índice & Caso Base & Caso 6 & Dif. (\%) \\
\hline LOLP $\left(\times 10^{2}\right)$ & 4,5472 & 4,5159 & $-0,69$ \\
\hline EENS $(\mathrm{GWh} / \mathrm{a})$ & 25,719 & 22,884 & $-11,02$ \\
\hline LOLF $($ oc. $/ \mathrm{a})$ & 152,82 & 149,39 & $-2,24$ \\
\hline LOLD $(\mathrm{h})$ & 2,5994 & 2,6409 & 1,60 \\
\hline LOLC $\left(10^{6} \mathrm{US} \$ / \mathrm{a}\right)$ & 45,060 & 40,716 & $-9,64$ \\
\hline
\end{tabular}




\section{CONCLUSÕES}

Este artigo apresentou uma aplicação da simulação Monte Carlo pseudo-cronológica na investigação dos efeitos do gerenciamento da demanda sobre o custo da perda de carga. O sistema Sul-Sudeste Brasileiro foi utilizado para testes, sendo avaliados os impactos obtidos pelo gerenciamento da carga da área Rio. Procurou-se enfatizar a necessidade de representar os diferentes padrões comportamentais da carga para as diversas áreas do sistema.

Através dos resultados apresentados, pode-se afirmar que as reduções no custo da perda de carga representam um aspecto altamente relevante para a análise de viabilidade de programas de gerenciamento da demanda.

\section{REFERÊNCIAS}

Anderberg, M. R., (1973). "Cluster Analysis for Applications", Academic Press, N. Y.

Billinton R. and Li, W., (1993). "A System State Transition Sampling Method for Composite System Reliability Evaluation", IEEE Transactions on Power Systems, Vol. 8, No. 3, pp. 761-770.

EPRI, (1989). "Customer demand for service reliability", REPORT RP-2810.

Gaul, A. J.; Handschin, E.; Hoffmann, W. And Lehmköster, C., (1998). "Establishing a Rule Base for a Hybrid ES/XPS Approach to Load Management", IEEE Transactions on Power Systems, Vol. 13, No. 1, pp. 86-93.

Gellings, C. W. And Smith, W. M., (1989). "Integrating Demand-Side Management into Utility Planning", IEEE Proceedings, Vol. 77, pp. 908-918.

Kumamoto, H.; Tanaka, K.; Inque, K. and Henley, E. J., (1980). "State Transition Monte Carlo for Evaluating Large Repairable Systems", IEEE Transactions on Reliability", Vol.R-29, No.5, pp.376-380.

Leite Da Silva, A. M.; Perez, A. G.; Marangon Lima, J. W. And Mello, J. C. O., (1997). "Loss of Load Costs in Generating Capacity Reliability Evaluation", Electric Power Systems Research, Vol. 41, pp. 109-116.

Leite da Silva, A. M.; Manso, L. A. F.; Mello J. C. O. and Billinton, R., (2000). "PseudoChronological Simulation for Composite Reliability Analysis with Time Varying Loads", IEEE Transactions on Power Systems, Vol. 15, No. 1, pp. 7380 .
Malik, A. S., (1998). "Simulation of DSM Resources as Generating Units in Probabilistic Production Costing Framework", IEEE Transactions on Power Systems, Vol. 13, No. 4, pp. 1528-1533.

Manso, L. A. F.; Leite Da Silva, A. M. and, Mello, J. C. O., (1998). "Comparação de Métodos Alternativos para Avaliação dos Custos de Perda de Carga em Sistemas de Geração e Transmissão", XII CBA, Uberlândia/MG, Vol. 6, pp.2291-2297.

Manso, L. A. F.; Leite da Silva, A. M. and Mello, J. C. O., (1999 a). "Comparison of Alternative Methods for Evaluating Loss of Load Costs in Generation and Transmission System", Electric Power Systems Research, Vol. 50, pp. 107-114.

Manso, L. A. F.; Leite da Silva, A. M. and Mello, J. C. O., (1999 b). "Avaliação da Confiabilidade de Sistemas de Geração e Transmissão Considerando Cargas Variantes no Tempo", XV SNPTEE, Foz do Iguaçu/PR, Grupo VII - GPL/11.

Massaud, A. G.; Hernandez, J. P. and Schilling, M. Th., (1992). "Custos de Interrupção de Energia Elétrica no Brasil", IX CBA, Vitória/ES, pp. 98-104.

Mello, J. C. O.; Pereira, M. V. F. and Leite da Silva, A. M., (1994). "Evaluation of Reliability Worth in Composite System Based on Pseudo-sequential Monte Carlo Simulation", IEEE Transactions on Power Systems, Vol. 9, No. 3, pp. 1318-1326.

Mello, J. C. O; Leite da Silva, A. M. and Pereira, M. V. F., (1997 a). "Efficient Loss-of-Load Cost Evaluation by Combined Pseudo-Sequential and State Transition Simulation", IEE Proc. C, Vol. 144, No. 2, pp. 147-154.

Mello, J. C. O.; Pereira, M. V. F.; Leite da Silva, A. M. and Melo, A. C. G., (1997 b). "Application of Chronological Load Modeling in Composite Reliability Worth Evaluation", Electric Power Systems Research, Vol. 40, pp. 167-174.

Melo, A. C. G.; Pereira, M. V. F. and Leite da Silva, A. M., (1993). "A Conditional Probability Approach to the Calculation of Frequency and Duration Indices in Composite Reliability Evaluation", IEEE Transactions on Power Systems, Vol. 8, No. 3, pp. $1118-1125$.

Sankarakrishnan, A. and Billinton, R., (1995). "Sequential Monte Carlo Simulation for Composite Power System Reliability Analysis with Time Varying Loads", IEEE Transactions on Power Systems, Vol. 10, No. 3, pp.1540-1545. 
Singh, C.; Pravin Chander, T. and Feng, J., (1993). "Convergence Characteristics of Two Monte Carlo Models for Reliability Evaluation of Interconnected Power Systems", Electric Power Systems Research, Vol. 28, No. 1, pp. 1-8.

Wacker, G. and Billinton, R., (1989). "Customer Cost of Electric Service Interruptions", Proc. IEEE, Vol. 77, No. 6, pp. 919-930. 